\title{
Nurse Led Smartphone Electrographic Monitoring for Atrial Fibrillation after Ischemic Stroke: SPOT-AF
}

\author{
Bernard Yan, ${ }^{\mathrm{a}}$ Hans Tu, ${ }^{\mathrm{b}}$ Christina Lam, ${ }^{\mathrm{a}}$ Corey Swift, ${ }^{\mathrm{a}}$ Ma Sze Ho, ${ }^{\mathrm{c}}$ Vincent C.T. Mok, ${ }^{\mathrm{c}}$ Yi Sui, ${ }^{\mathrm{d}}$ \\ David Sharpe, ${ }^{\mathrm{e}}$ Darshan Ghia, ${ }^{\mathrm{f}}$ Jim Jannes, ${ }^{\mathrm{g}}$ Stephen Davis, ${ }^{\mathrm{a}}$ Xinfeng Liu, ${ }^{\mathrm{h}}$ Ben Freedman ${ }^{\mathrm{i}}$ \\ aThe Melbourne Brain Centre at the Royal Melbourne Hospital and the University of Melbourne, Parkville, Australia \\ ${ }^{b}$ Department of Neurology and Medicine, Western Health, The University of Melbourne, Footscray, Australia \\ 'Lui Che Woo Institute of Innovative Medicine, Therese Pei Fong Chow Research Centre for Prevention of Dementia, Prince of Wales Hospital, \\ and Department of Medicine and Therapeutics, The Chinese University of Hong Kong, Hong Kong, China \\ ${ }^{d}$ Department of Neurology, Shenyang First People's Hospital, Shenyang, China \\ eNeurology Department, Concord General Hospital, Sydney, Australia \\ ${ }^{f}$ Fiona Stanley Hospital and University of Western Australia, Perth, Australia \\ ${ }^{9}$ Department of Neurology, Royal Adelaide Hospital, Adelaide, Australia

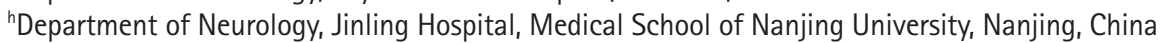 \\ 'Heart Research Institute Charles Perkins Centre, and Concord Hospital Cardiology, University of Sydney, Sydney, Australia
}

Background and Purpose Paroxysmal atrial fibrillation (PAF) underlying acute stroke frequently evades detection by standard practice, considered to be a combination of routine electrocardiogram (ECG) monitoring, and 24-hour Holter recordings. We hypothesized that nurse-led in-hospital intermittent monitoring approach would increase PAF detection rate.

Methods We recruited patients hospitalised for stroke/transient ischemic attack, without history of atrial fibrillation (AF), in a prospective multi-centre observational study. Patients were monitored using a smartphone-enabled handheld ECG (iECG) during routine nursing observations, and underwent 24-hour Holter monitoring according to local practice. The primary outcome was comparison of AF detection by nurse-led iECG versus Holter monitoring in patients who received both tests: secondary outcome was oral anticoagulant commencement at 3-month following PAF detection.

Results One thousand and seventy-nine patients underwent iECG monitoring: 294 had iECG and Holter monitoring. AF was detected in $25 / 294$ (8.5\%) by iECG, and $8 / 294(2.8 \%)$ by 24 -hour Holter recordings $(P<0.001)$. Median duration from stroke onset to AF detection for iECG was 3 days (interquartile range [IQR], 2 to 6$)$ compared with 7 days (IQR, 6 to 10) for Holter recordings $(P=0.02)$. Of 25 patients with AF detected by $\mathrm{EECG}, 11$ were commenced on oral anticoagulant, compared to $5 / 8$ for Holter. AF was detected in $8.8 \%$ (69/785 patients) who underwent iECG recordings only ( $P=0.8$ vs. those who had both $\mathrm{EECG}$ and 24 -hour Holter).

Conclusions Nurse-led in-hospital iECG surveillance after stroke is feasible and effective and detects more PAF earlier and more frequently than routine 24-hour Holter recordings. Screening with iECG could be incorporated into routine post-stroke nursing observations to increase diagnosis of PAF, and facilitate institution of guideline-recommended anticoagulation.
Correspondence: Ben Freedman Heart Research Institute Charles Perkins Centre, University of Sydney, Sydney 2006, Australia Tel: +61-2-9114-2199 Fax: +61-2-8212-9058 E-mail: ben.freedman@sydney.edu.au https://orcid.org/0000-0002-38092911

Co-correspondence: Xinfeng Liu Department of Neurology, Jinling Hospital, Medical School of Nanjing University, 305 East Zhongshan Road, Xuanwu, Nanjing, China Tel: +86-2584664563 Fax: +86-13813835114 E-mail:xfliu2@vip.163.com

Received: March 4, 2020

Revised: June 16, 2020

Accepted: June 17, 2020

Keywords Atrial fibrillation; Stroke; Smartphone; Electrocardiography 


\section{Introduction}

In patients presenting with ischemic stroke or transient ischemic attack (TIA), nearly a quarter have underlying atrial fibrillation (AF). ${ }^{1}$ However, AF is often paroxysmal and can evade detection by a single time point 12-lead electrocardiogram (ECG) in up to $48 \%{ }^{2}$ Following stroke, the incidence of AF on 12-lead ECG is between $2 \%$ and $15 \% .{ }^{1-3}$ Although useful, 24-hour Holter monitoring detects AF in only 1\% to 6\% (mean 2.9\%). ${ }^{4-6}$ Despite guidelines recommending at least 24 hours cardiac monitoring post-stroke, ${ }^{7,8}$ adherence to Holter monitoring is relatively infrequent in the real world. The Ontario Stroke Registry published in 2016 found that only 30\% of patients presenting with first acute ischemic stroke or TIA had 24-hour of Holter monitoring within 30 days and $<1 \%$ of patients received $>48$ hours of cardiac monitoring within 90 days of symptom onset. ${ }^{9}$

Longer term cardiac monitoring devices including implantable cardiac monitors (ICM), event-triggered recorders and ECG monitoring patches are more sensitive alternatives to Holter monitoring. The AF detection rates with ICM vary between $9 \%$ and $25 \%$ at 6 months ${ }^{10-12}$ while a 30-day event recorder detected AF in 12\% to $16 \%$ of patients. ${ }^{13,14}$ Although long-term implanted monitoring devices increase AF detection rates, their use may be limited by costs, and concerns for adverse events which include $2.4 \%$ incidence of pocket infection or erosion, 1.4\% pain and $1.9 \%$ irritation or inflammation. ${ }^{10}$ Cost of device and the implantation procedure is also a major limitation, particularly in resource restricted countries. ECG monitoring patches with a 14day capability are an alternative, but occasionally cause dermatological irritation from skin surface electrodes $(2.3 \%){ }_{1}^{15}$ and are also expensive.

A smartphone-enabled handheld ECG device (iECG), AliveCor (AliveCor, San Francisco, CA, USA), may potentially overcome these limitations. The iECG is a palm-sized device which records a 30-second single channel lead 1 ECG rhythm strip, and allows users to view the ECG rhythm strips in real-time. The inbuilt algorithm makes an automated diagnosis of "normal," "possible AF," or "unclassified." It has been used in community opportunistic screening (Screening, Education And Recognition in Community pHarmacies of Atrial Fibrillation [SEARCH-AF] study ${ }^{16}$ ), and with patient-initiated recordings over 1 year in the assessment of REmote HEArt Rhythm sampling using the AliveCor Heart Monitor to scrEen for Atrial Fibrillation (REHEARSE-AF) study. ${ }^{17}$ The AliveCor iECG has sensitivity of $98 \%$ and specificity of $97 \%$ for AF diagnosis compared to 12-lead ECG, interpreted by cardiologists. ${ }^{18}$ We hypothesized that an iECG could be readily incorporated into routine stroke unit nursing observations in patients admitted to hospital after a stroke or TIA, and would be an effective and inexpensive way to increase detection of paroxysmal atrial fibrillation (PAF).

The aim of our study was to compare the detection rates of PAF by a pragmatic strategy of nurse-led intermittent iECG recordings during routine clinical observations, with the current standard 24-hour Holter monitoring where available in an international patient cohort hospitalised in a stroke unit with acute ischemic stroke or TIA.

\section{Methods}

This was a pragmatic observational, multi-centre study with eight participating centres, including Royal Melbourne Hospital and Western Hospital (both Melbourne, Victoria); Royal Adelaide Hospital (Adelaide, South Australia); Fiona Stanley Hospital (Perth, Western Australia); Concord Hospital (Sydney NSW), Jinling Hospital (Nanjing, China), Shenyang First People's Hospital (Shenyang, China), and Prince of Wales Hospital (Hong Kong).

\section{Patient population}

Patients were eligible for enrolment if they presented with

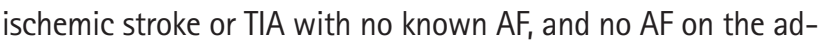
mission 12-lead ECG. Patients were excluded if the treating medical team considered long-term oral anticoagulation use inappropriate because the stroke was very severe, or in the light of other co-morbidities.

\section{iECG heart monitor}

The AliveCor Kardia Mobile is a wireless handheld device with two metal electrodes. The device records and transmits a 30-second single lead 1 ECG to the smartphone microphone using frequency-modulated ultrasound signals, when fingers of both hands are placed on the electrodes (Figure 1). The ECG signal received by the smartphone microphone is digitized to an ECG trace (300 samples/s, 16bit resolution). The lead 1 ECG rhythm strip can be viewed in real-time or stored, and is instantly transmitted by the smart-phone to a secure server which is Health Insurance Portability and Accountability Act (US HIPAA) confidentiality/privacy standards compliant. The file is stored as a PDF file for physician interpretation accessible through a passwordprotected website. An automated algorithm in the application produces noise-filtered traces for viewing on the smartphone, and $\mathrm{AF}$ diagnosis based on criteria of $\mathrm{p}$-wave absence and $\mathrm{R}-\mathrm{R}$ interval irregularity (Figure 2). The AliveCor has Conformité Européenne (CE) mark, and is approved by the Food and Drug Administration and the Australian Therapeutic Goods Administration for use as a medical device. 


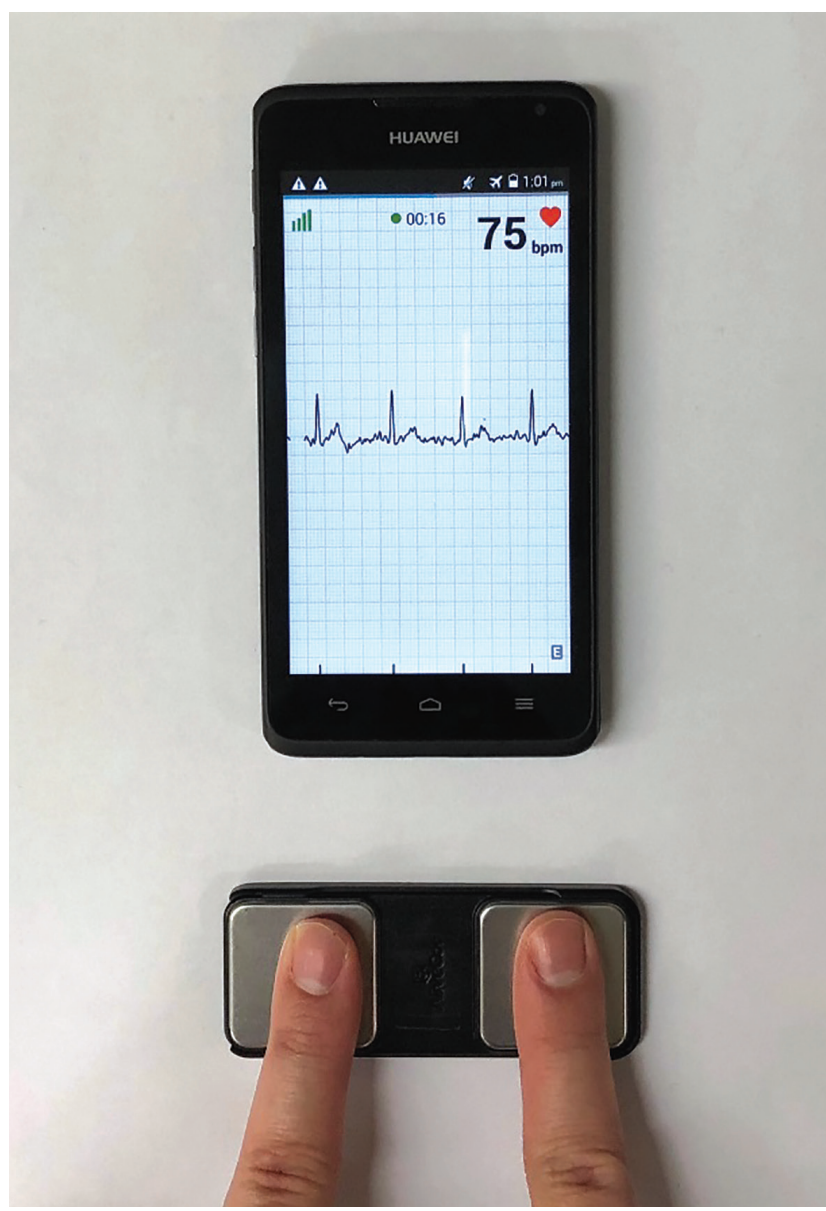

Figure 1. AliveCor Kardia mobile electrocardiogram (ECG) device, showing index finger of right and left hand touching the respective electrode. Recorded ECG rhythm strip shows sinus rhythm.

\section{Monitoring protocol}

From 2015 to 2018, patients presenting to participating acute stroke units were screened to determine their eligibility for participation. Consecutive eligible patients underwent intermittent hand-held iECG recordings that were performed by nursing staff trained to use the device on patients during routine nursing vital sign observations (typically every 2 to 4 hours) at the participating stroke units, until discharge. For each monitoring session, the device was applied for 30 seconds, which is the duration required by the iECG device. All patients received in-patient 12lead ECG. Patients also underwent in-patient or out-patient Holter monitoring at the discretion of the treating stoke team, according to their usual practice. AF was defined as episodes lasting 30 seconds or longer. All 'possible AF' iECG recordings were reviewed immediately in real time by qualified physician investigators at each site. In the event of uncertainty ( $n=15$ iECG traces), the iECG rhythm trace was examined by another experienced physician and a consensus diagnosis reached (B.Y., H.T., B.F.). The qualified physicians are those who have complet-

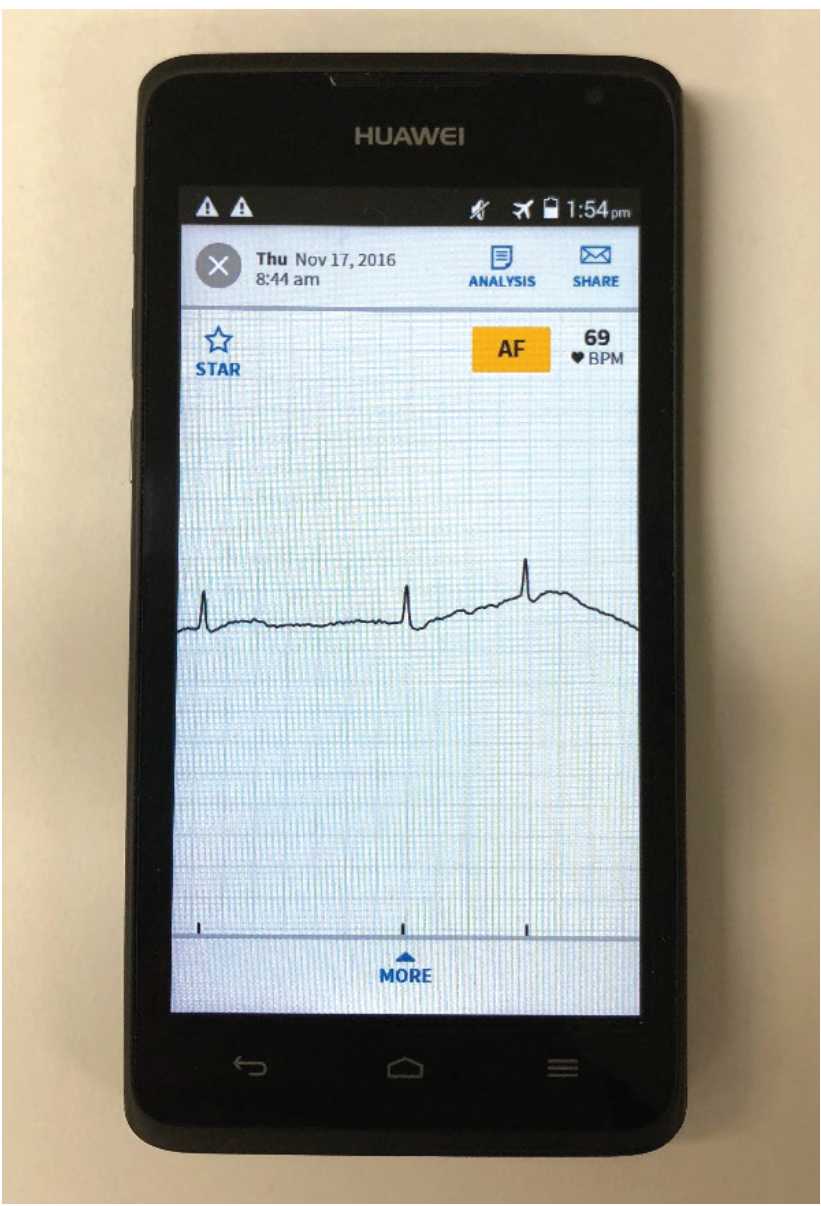

Figure 2. Smartphone-enabled handheld ECG (iECG) showing slow atrial fibrillation, with automated diagnosis of possible atrial fibrillation.

ed physician training with compulsory proficiency in ECG assessment and also trained cardiologists and vascular neurologists. The management of AF diagnosed during monitoring was at the discretion of the treating team at the participating stroke units.

\section{Clinical data}

Patients' baseline characteristics including age, sex, and $\mathrm{CHA}_{2} \mathrm{DS}_{2}$-VASc scores were recorded on a clinical record form, as were stroke onset date/time, and arrival date/time to hospital, Oxfordshire score and antithrombotic medications. Based on clinical features and imaging, patients were classified into either total anterior circulation syndrome (TACS), partial anterior circulation syndrome (PACS), lacunar syndrome (LACS), or posterior circulation syndrome. AF diagnoses made as a result of the study, and anticoagulant use at 3-month were also recorded.

\section{Primary and secondary outcomes}

The outcome endpoints are presented below. ${ }^{19}$ 


\section{Primary outcomes}

(1) Proportion of new AF detected using iECG recordings compared to Holter monitoring, in the subset of patients who received both investigations.

(2) Proportion of patients with new AF detected using nurseled iECG recordings.

\section{Secondary outcomes}

(1) Time from stroke onset to AF detection for each monitoring method.

(2) Proportion of patients anticoagulated at 3 months followup for each monitoring method and all methods combined for newly diagnosed $\mathrm{AF}$.

\section{Statistical analysis}

Continuous variables are reported as median with interquartile range (IQR) and analysed using Wilcoxon rank sum test. Categorical variables are described as percentages and analysed by chi-square tests. Comparisons of the proportion of patients with AF detected on $\mathrm{iECG}$ versus Holter monitoring was performed using the McNemar's test. Analyses were conducted using STATA 15IC (StataCorp., College Station, TX, USA) and SPSS version 20.0.0 (IBM Co., Armonk, NY, USA).

\section{Ethical considerations}

Ethics approval for this study was attained with patient consent waiver through the Melbourne Health Human Research Ethics Committee on 25 March 2015. Additionally, this study received ethical approval from relevant Institutional Review Boards for participating centres in China, Hong Kong, South Australia, New South Wales and Western Australia.

The steering committee comprising the authors monitored the study implementation, data collection, and analyses. AliveCor Inc. (San Francisco, CA, USA) provided a limited supply of AliveCor Kardia monitors for use in this study.

\section{Results}

\section{Patient characteristics}

During the study period, 1,079 participants were recruited into the study from the eight participating hospitals and all underwent nurse-led iECG recordings. The baseline characteristics of the patients are shown in Table 1. The median age was 66 years (IQR, 55 to 75$), 61 \%$ were men and median $\mathrm{CHA}_{2} \mathrm{DS}_{2}$-VASc score was 4 (IOR, 3 to 5). Of 1,079 participants, 2.2\% had congestive heart failure, 64\% had hypertension and 25\% had diabetes. The median days from stroke onset to hospital visit was $0(I Q R, 0$ to 1$)$.

A total of 294 patients (27\%) also underwent Holter monitoring and were included in the primary analysis (Figure 3). Their median age was 68 years (IQR, 57 to 77), 54\% were men, and median $\mathrm{CHA}_{2} \mathrm{DS}_{2}$-VASc score was 4 (IQR, 3 to 5$) ; 2.7 \%$ had congestive heart failure, 59\% had hypertension and $28 \%$ had diabetes. Baseline characteristics for the 294 patients are summarised in Table 1. Of the 294 patients who underwent both 24-hour Holter and iECG monitoring, two did not provide their age, and five had missing Oxfordshire score. Forty-one (14\%) were lost to follow-up and two died before 3 months.

As shown in Table 1 there were some differences between pa-

Table 1. Patient characteristics

\begin{tabular}{|c|c|c|c|c|}
\hline Characteristic & Whole group ${ }^{*}(n=1,079)$ & Holter $(n=294)^{+}$ & No Holter ${ }^{\ddagger}(n=785)$ & $P$ \\
\hline Age (yr) & $66(55-75)$ & $68(57-77)$ & $64(54-75)$ & 0.005 \\
\hline Male sex & $663(61.4)$ & $159(54.1)$ & $504(64.2)$ & 0.002 \\
\hline $\mathrm{CHA}_{2} \mathrm{DS}_{2}$-VASc & $4(3-5)$ & $4(3-5)$ & $3(3-5)$ & 0.061 \\
\hline Congestive heart failure & $24(2.2)$ & $8(2.7)$ & $16(2.0)$ & 0.500 \\
\hline Hypertension & $691(64)$ & $174(59.4)$ & $517(65.9)$ & 0.039 \\
\hline Diabetes & $265(24.6)$ & $83(28.2)$ & $182(23.2)$ & 0.088 \\
\hline Oxford & & & & $<0.001$ \\
\hline TACS & $136(12.6)$ & $45(15)$ & $91(11)$ & 0.114 \\
\hline PACS & $537(49.8)$ & $119(40)$ & 418 (53.2) & $<0.001$ \\
\hline POCS & $208(19.3)$ & $57(19.4)$ & $151(19.2)$ & 0.988 \\
\hline LACS & $144(13)$ & $65(22.1)$ & $79(10)$ & $<0.001$ \\
\hline TIA & $28(2.6)$ & $3(1.0)$ & $25(3.2)$ & 0.044 \\
\hline
\end{tabular}

Values are presented as median (interquartile range) or number (\%).

TACS, total anterior circulation syndrome; PACS, partial anterior circulation syndrome; POCS, posterior circulation syndrome; LACS, lacunar syndrome; TIA, transient ischemic attack.

*Whole group: 5 patients were missing for age, 3 for sex and 26 for 0 xfordshire score; ${ }^{+}$Holter: 2 patients missing for age, 5 missing for $0 x f o r d ;{ }^{*}$ No holter: 3 missing for age, 3 missing for sex and 21 missing for Oxfordshire score. 


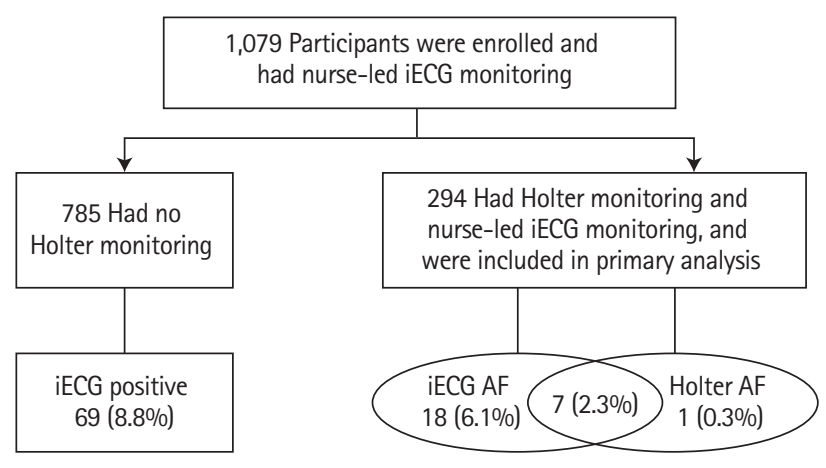

Figure 3. Patient flow diagram. iECG, smartphone-enabled handheld ECG; $A F$, atrial fibrillation.

tients who received Holter monitoring and those who did not. Patients who received Holter monitoring were slightly older, and a lower proportion were male. Fewer had PACS, and more had LACS strokes. There were also slight differences between patients who were iECG AF positive and those who were negative. Patients who were iECG positive were older, had a higher $\mathrm{CHAD}_{2} \mathrm{DS}_{2}$-VASc score and fewer had LACS strokes (Table 2).

\section{Primary and secondary outcomes AF detection}

The nurse-led iECG recordings detected AF in 25 (8.5\%) patients, while 24-hour Holter monitoring detected AF in eight (2.8\%). AF detection rate by nurse-led iECG recordings was significantly greater than Holter monitoring (McNemar test $X^{2}=15.21$, $P<0.001)$. Of the eight patients found to have AF on Holter monitoring, seven also had AF on the nurse-led iECG recordings.
Conversely, of the 25 patients who had AF detected on nurse-led iECG recordings, Holter monitoring detected AF in only seven. The kappa value for agreement between the iECG and Holter was 0.40 .

For patients detected to have AF on $\mathrm{EECG}(\mathrm{n}=25)$, the median number of days monitored was $4(I Q R, 4$ to 6$)$ and median number of recordings was 10 (IQR, 6 to 12). AF was detected significantly earlier by iECG recordings, at a median of 3 days from stroke onset (IQR, 2 to 6) than for the eight patients who had AF detected by Holter monitoring, in whom AF was detected at a median of 7 days after stroke (IQR, 6 to 10; $P=0.02$ ). Of the 294 patients who did not have AF detected on $\mathrm{EECG}(\mathrm{n}=269)$ the median number of days monitored was three $(I Q R, 2$ to 5$)$ and the median number of recordings was five (IQR, 2 to 8 ).

In the overall group of 1,079 patients the median number of days monitored was four (IQR, 3 to 5 ) and median number of recordings was 12 (IQR, 4 to 12). AF was detected by nurse-led iECG recordings in 94/1,079 (8.7\%) patients at a median of 4 days after stroke onset (IQR, 2 to 6$)$, the median number of days monitored was four (IQR, 4 to 5 ), and median number of recordings was 12 (IQR, 6 to 12), the number of recordings and days monitored were identical in the 985 patients where AF was not identified by iECG. To demonstrate the absence of bias, we evaluated the proportion who underwent Holter monitoring in both $\mathrm{iECG}$ positive for AF and $\mathrm{BECG}$ negative for AF. Of the 94 iECG AF positive patients, 25/94 (27\%) underwent Holter monitoring which was identical to the group with no $\mathrm{AF}$ identified on iECG (269/985, 27\%).

For the 785 patients who underwent iECG recording only, AF

Table 2. Patient characteristics for iECG AF positive and negative

\begin{tabular}{|c|c|c|c|}
\hline Characteristic & AF positive* iECG $(n=94)$ & AF negative ${ }^{+}$iECG $(n=985)$ & $P$ \\
\hline Age $(y r)$ & $74.5(65-80)$ & $64(61-80)$ & $<0.001$ \\
\hline Male sex & $53(57.6)$ & $611(62)$ & 0.39 \\
\hline $\mathrm{CHA}_{2} \mathrm{DS}_{2}$-VASc & $4.19 \pm 1.29$ & $3.66 \pm 1.26$ & $<0.001$ \\
\hline Congestive heart failure & $3(3.1)$ & $21(2.1)$ & 0.50 \\
\hline Hypertension & $63(67)$ & $628(63.7)$ & 0.54 \\
\hline Diabetes & $21(22.3)$ & $244(28 \cdot 2)$ & 0.59 \\
\hline \multicolumn{4}{|l|}{ Oxford } \\
\hline TACS & $16(17.2)$ & $120(12.5)$ & 0.25 \\
\hline PACS & $51(54.8)$ & $486(50.6)$ & 0.50 \\
\hline POCS & $19(20.4)$ & 189 (19.7) & 0.97 \\
\hline LACS & $5(5.4)$ & 139 (14.4) & 0.02 \\
\hline TIA & $2(2.1)$ & $26(2.7)$ & 0.74 \\
\hline
\end{tabular}

Values are presented as median (interquartile range), number (\%), or mean \pm standard deviation.

iECG, smartphone-enabled handheld ECG; AF, atrial fibrillation; TACS, total anterior circulation syndrome; PACS, partial anterior circulation syndrome; POCS, posterior circulation syndrome; LACS, lacunar syndrome; TIA, transient ischemic attack.

${ }^{*} \mathrm{AF}$ positive: 2 missing for sex and 1 missing for Oxfordshire score; ${ }^{\dagger} \mathrm{AF}$ negative: 5 missing for age, 1 missing for sex and 25 missing for 0 xfordshire score. 
was detected in 69 (8.8\%). The AF detection rate did not differ from those who had both iECG and 24-hour Holter monitoring $(8.5 \%, P=0.8)$. The median days monitored from stroke onset to AF detection was 4 days for the subset who underwent iECG recordings only (IQR, 2 to 6$)$, which did not differ from those who had both iECG and Holter recordings ( 3 days; IQR, 2 to $6 ; P=0.7$ ). The proportion of patients who also received Holter monitoring was higher in the Australian centres (160/429 patients, 37\%), than in the centres in China $(134 / 650,20 \%, P<0.001)$.

\section{Anticoagulation therapy}

At 3 months post-stroke, five of the eight patients detected to have AF on Holter monitoring, had been commenced on anticoagulation therapy, including four of seven patients with AF detection by both methods. A similar proportion of those with AF detected by the nurse-led IECG recordings $(11 / 25)$ had been commenced on anticoagulation therapy $(P=0.4)$, though numbers are small. Among patients who did not receive Holter monitoring, those with AF detected on the nurse-led iECG recordings ( $n=69), 25(36 \%)$ had been commenced on an anticoagulant at 3 months.

The 3-month anticoagulation rate for patients with AF detected by the nurse-led iECG recordings in the Australian centres was $43 \%$ (16 of 37) which was higher than in centres in China $(20 / 57,35 \%)$, but this difference was not significant $(P=0.4)$.

\section{Discussion}

Our study is the first to compare a novel strategy of nurse-led AF detection using an iECG incorporated into routine observations, with the current standard of care, 24-hour Holter monitoring, in the post-acute stroke setting in hospital. We showed that this strategy after ischemic stroke or TIA, identified new AF in significantly more patients (8.5\%) than 24-hour Holter monitoring, which identified AF in only $2.8 \%$, and identified AF earlier. Intermittent patient-activated recordings using a similar handheld ECG device in 249 patients within 2 weeks of an acute ischemic stroke found a $6 \%$ incidence of new AF compared to only $2 \%$ with a 24-hour Holter recorder, ${ }_{1}^{20}$ a similar differential for intermittent monitoring versus 24-hour continuous Holter monitoring. The AF detection rate by iECG in our study was higher than in other studies of $\mathrm{EECG}$ recordings using similar technology in primary care or populations selected for older age ${ }_{1}^{16,17,21}$ which is not surprising given we used multiple recordings in patients with acute stroke or TIA.

Only 294/1,079 patients (27\%) underwent 24-hour Holter monitoring. We attributed the low Holter monitoring rate to the pragmatic nature of the study which reflects usual practice. Although we encouraged investigators to perform Holter monitoring, the decision to undertake cardiac investigations was left at the discretion of the local physicians. Nevertheless, this rate of Holter monitoring is in line with previous studies which investigated real-world practice of post-stroke investigations. Edwards et al. ${ }_{1}^{9}$ showed that among 17,398 patients, 20\% had 24-hour Holter monitoring within the first week, increasing to almost $30 \%$ within 30 days. That study was performed in Canada and was representative of routine practice in that country. This contrasted with a slightly higher rate of Holter monitoring of 40\% of patients with embolic stroke of undetermined source. ${ }^{22} \mathrm{~A}$ significant proportion of our patients were recruited from Asian centres (60\%), compared with $21 \%$ in the study by Perera et al. ${ }^{22}$ We postulated that the routine practice of physicians in Asia was informed by a perception of lower prevalence of $\mathrm{AF}_{1}^{23,24}$ leading to reluctance in initiating post-stroke cardiac investigations, and a lower rate of Holter monitoring as occurred in the centres from China in our study.

The ideal method and timing of monitoring for AF has not been elucidated. ${ }^{25}$ Obviously, longer and more continuous monitoring is performed will result in higher AF detection rate. But more intensive monitoring is more expensive and more difficult to implement in many health systems. In previous studies, e.g., FIND-AF (Finding atrial fibrillation in stroke- evaluation of enhanced and prolonged Holter ECG) two-thirds of patients eventually diagnosed with AF by repeated 10-day Holter monitoring over 6 months reached the diagnosis in the first 10-day recording, commenced at a median of 4 days after stroke onset. ${ }^{6}$ Using implanted cardiac monitors, Ziegler et al. ${ }^{11}$ found at 30-day the rate of $\mathrm{AF}$ was $4.6 \%$, and at 6 months, $12.2 \%$, while Flint et al., ${ }^{13}$ found that a 30-day external event loop recorder detected $45 \%$ of AF in the first 10 days. Poulsen et al. ${ }^{26}$ detected a higher proportion of AF (21\%) using a patient-activated hand-held Zenicor device, although patients were monitored for 30-days in that study, in comparison to our study, where the median number of days monitored was 4 . Clearly, early monitoring is important for the detection of $A F$, and that is a major advantage of the strategy we devised. While limiting detection to a finite window may underestimate late PAF, the immediate gains are valuable.

Only 44\% of patients diagnosed with AF on iECG recordings in hospital were commenced on anticoagulation therapy. The number of patients with AF was too small to determine if there was any difference between the two strategies in anticoagulation rate. The modest rate of commencement of secondary anticoagulant prevention regrettably reflects real world practice, which does not closely adhere to American Guidelines. ${ }^{7}$ There was a non-significant trend ( $43 \%$ vs. $35 \%$ ) towards a higher 
anticoagulant rate in Australia than in China and Hong Kong. In a retrospective study investigating anticoagulation use after ischemic stroke in 14 hospitals in Northwestern China, only $20 \%$ of patients diagnosed with AF were commenced on anticoagulants. ${ }^{27}$ In Australia, Pandya et al. ${ }^{28}$ showed that $52 \%$ of patients with AF were commenced on an anticoagulant, while in Germany, a 2015 study reported only 18\% commencement of anticoagulation therapy. ${ }^{29}$ The lower anticoagulation use in China may also result from the high cost of the non-vitamin $\mathrm{K}$ dependent oral anticoagulants (NOACs), which are unaffordable to many patients. ${ }^{30}$ Moreover, lower warfarin use may be related to a perceived higher bleeding risk and lack of access to international normalized ratio (INR) testing. ${ }^{31}$

Our study findings have important implications for clinical practice. Given the low rate of AF detection by single episode admission ECGs and the low usage of Holter monitoring in stroke patients post-discharge, the great advantage of smartphone-enabled cardiac monitoring by nurses is its ready availability during the hospital period, even in resource-restricted health systems. Furthermore, it is inexpensive and required only basic training of the nurses. It can also be used early after stroke where AF is more likely to occur, potentially increasing the proportion of patients who are monitored for AF and detected with the arrhythmia in a timely fashion. The automated algorithm also permits expeditious AF diagnosis without requirement for immediate specialist interpretation of ECG traces, while still allowing for easy specialist over-read. We envisage that there would be few barriers to implement smartphone-enabled cardiac monitoring by nurses in hospital stroke units.

Our study has several limitations. Firstly, Holter and nurseled iECG monitoring were not performed simultaneously, because the iECG recordings were restricted to inpatient care, while Holter monitoring was performed either as an inpatient or outpatient according to usual practice. However, our aim was to compare an inexpensive nurse-led strategy with routine Holter monitoring according to usual practice in each stroke unit, and was thus a pragmatic study comparing the new strategy with usual care. Secondly, only a quarter of patients had both 24-hour Holter and iECG recordings, but this reflects usual practice in those stroke units, who would have received no additional monitoring for AF. Moreover, patients diagnosed with AF on IECG may have influenced the decision to additionally use Holter monitoring. This could lead to a selection bias of patients with lower odds of being diagnosed with AF in the Holter group. However, the proportion of patients who received Holter monitoring was identical in both iECG AF positive and negative groups and likewise the proportion of AF detected on iECG for the Holter and non-Holter group did not differ. It is possible that some patients with PAF were not identified given we screened patients only during hospitalization. Screening patients in the outpatient setting by patient-initiated intermittent recordings, long-term Holter monitoring, 14 day patches, or even implanted devices would identify additional patients with PAF who were not detected while in hospital, but these strategies are neither routinely adopted in many countries nor recommended in many guidelines. In our study, Holter monitoring was performed for a period of 24 hours. Stahrenberg et al., ${ }^{3}$ showed that prolonged monitoring (7 days) detected more AF than standard of care (24 hours), $12.5 \%$ and $4.8 \%$ respectively. On this basis, we conceded that monitoring for $>24$ hours by Holter monitoring have detected more cases of AF.

The approximate annual number of stroke patients for each participating hospital is estimated to be between 800 and 1,000 . Recruitment of patients was limited according to the inclusion and exclusion criteria. Site commencement in the study and duration of patient recruitment varied between the sites, and was staggered throughout the 3 years, with some sites not commencing recruitment until the third year. Finally, although the iECG has a specificity of $97 \%$, it is possible the iECG yielded false positive AF results, and only iECGs verified by the attending physicians were included as positive. Any uncertain AF traces following review by the attending physician were reviewed by three experienced physicians.

\section{Conclusions}

We showed that a nurse-led strategy of intermittent hand-held iECG recordings as part of collection of routine vital signs in the post-stroke period in hospital detected new PAF in 8.5\% of patients, significantly more, and significantly earlier post-stroke than routine 24-hour Holter monitoring performed in only 27\% of patients. Nurse-led smartphone-enabled intermittent cardiac monitoring with an IECG could be considered complementary to standard routine post-stroke investigations for AF or could even replace routine 24-hour Holter monitoring as a scalable lowcost solution which could be widely implemented in countries with diverse availability of health resources to increase the yield of AF detection early after stroke or TIA.

\section{Disclosure}

This study was supported by a small grant from Boehringer Ingelheim. 


\section{Acknowledgments}

The authors would like to acknowledge and thank the nursing staff for their assistance with the iECG monitoring of stroke patients. The authors would also like to thank RMH Neuroscience Foundation for the support.

\section{References}

1. Sposato LA, Cipriano LE, Saposnik G, Ruíz Vargas E, Riccio PM, Hachinski V. Diagnosis of atrial fibrillation after stroke and transient ischaemic attack: a systematic review and meta-analysis. Lancet Neurol 2015;14:377-387.

2. Rizos $T$, Wagner $A$, Jenetzky $E$, Ringleb $P A$, Becker $R$, Hacke $W$, et al. Paroxysmal atrial fibrillation is more prevalent than persistent atrial fibrillation in acute stroke and transient ischemic attack patients. Cerebrovasc Dis 2011;32:276-282.

3. Stahrenberg R, Weber-Krüger M, Seegers J, Edelmann F, Lahno $R$, Haase $B$, et al. Enhanced detection of paroxysmal atrial fibrillation by early and prolonged continuous holter monitoring in patients with cerebral ischemia presenting in sinus rhythm. Stroke 2010;41:2884-2888.

4. Schnabel RB, Haeusler KG, Healey JS, Freedman B, Boriani G, Brachmann J, et al. Searching for atrial fibrillation poststroke: a white paper of the AF-SCREEN International Collaboration. Circulation 2019;140:1834-1850.

5. Lazzaro MA, Krishnan K, Prabhakaran S. Detection of atrial fibrillation with concurrent Holter monitoring and continuous cardiac telemetry following ischemic stroke and transient ischemic attack. J Stroke Cerebrovasc Dis 2012;21:89-93.

6. Wachter R, Gröschel K, Gelbrich G, Hamann GF, Kermer P, Liman $J$, et al. Holter-electrocardiogram-monitoring in patients

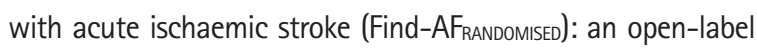
randomised controlled trial. Lancet Neurol 2017;16:282-290.

7. Powers WJ, Rabinstein AA, Ackerson T, Adeoye OM, Bambakidis NC, Becker K, et al. 2018 Guidelines for the early management of patients with acute ischemic stroke: a guideline for healthcare professionals from the American Heart Association/American Stroke Association. Stroke 2018;49:e46-e110.

8. Coutts $S B$, Wein $T H$, Lindsay MP, Buck $B$, Cote $R$, Ellis $P$, et al. Canadian Stroke Best Practice Recommendations: secondary prevention of stroke guidelines, update 2014. Int J Stroke 2015;10:282-291.

9. Edwards JD, Kapral MK, Fang J, Saposnik G, Gladstone DJ; Investigators of the Registry of the Canadian Stroke Network. Underutilization of ambulatory ECG monitoring after stroke and transient ischemic attack: missed opportunities for atrial fibrillation detection. Stroke 2016;47:1982-1989.

10. Sanna T, Diener HC, Passman RS, Di Lazzaro V, Bernstein RA, Morillo CA, et al. Cryptogenic stroke and underlying atrial fibrillation. N Engl J Med 2014;370:2478-2486.

11. Ziegler PD, Rogers JD, Ferreira SW, Nichols AJ, Richards $M$, Koehler JL, et al. Long-term detection of atrial fibrillation with insertable cardiac monitors in a real-world cryptogenic stroke population. Int J Cardiol 2017;244:175-179.

12. Carrazco C, Golyan D, Kahen M, Black K, Libman RB, Katz JM. Prevalence and risk factors for paroxysmal atrial fibrillation and flutter detection after cryptogenic ischemic stroke. $J$ Stroke Cerebrovasc Dis 2018;27:203-209.

13. Flint $A C$, Banki NM, Ren $X$, Rao VA, Go AS. Detection of paroxysmal atrial fibrillation by 30-day event monitoring in cryptogenic ischemic stroke: the Stroke and Monitoring for PAF in Real Time (SMART) Registry. Stroke 2012;43:27882790.

14. Gladstone DJ, Spring M, Dorian P, Panzov V, Thorpe KE, Hall $J$, et al. Atrial fibrillation in patients with cryptogenic stroke. N Eng/ J Med 2014;370:2467-2477.

15. Steinhubl SR, Waalen J, Edwards AM, Ariniello LM, Mehta RR, Ebner GS, et al. Effect of a home-based wearable continuous ECG monitoring patch on detection of undiagnosed atrial fibrillation: the mSToPS randomized clinical trial. JAMA 2018;320:146-155.

16. Lowres N, Neubeck L, Salkeld G, Krass I, McLachlan AJ, Redfern $J$, et al. Feasibility and cost-effectiveness of stroke prevention through community screening for atrial fibrillation using iPhone ECG in pharmacies: the SEARCH-AF study. Thromb Haemost 2014;111:1167-1176.

17. Halcox JPJ, Wareham K, Cardew A, Gilmore M, Barry JP, Phillips

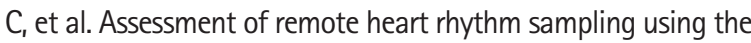
AliveCor Heart Monitor to screen for atrial fibrillation: the REHEARSE-AF study. Circulation 2017;136:1784-1794.

18. Lau JK, Lowres N, Neubeck L, Brieger DB, Sy RW, Galloway $C D$, et al. iPhone ECG application for community screening to detect silent atrial fibrillation: a novel technology to prevent stroke. Int J Cardiol 2013;165:193-194.

19. Tu HT, Chen Z, Swift C, Churilov L, Guo R, Liu X, et al. Smartphone electrographic monitoring for atrial fibrillation in acute ischemic stroke and transient ischemic attack. Int J Stroke 2017;12:786-789.

20. Doliwa Sobocinski P, Anggårdh Rooth $E$, Frykman Kull V, von Arbin $M$, Wallén $H$, Rosenqvist $M$. Improved screening for silent atrial fibrillation after ischaemic stroke. Europace 2012;14:1112-1116.

21. Orchard J, Lowres N, Freedman SB, Ladak L, Lee W, Zwar N, et al. Screening for atrial fibrillation during influenza vaccina- 
tions by primary care nurses using a smartphone electrocardiograph (iECG): a feasibility study. Eur J Prev Cardiol 2016;23(2 Suppl): 13-20.

22. Perera KS, Vanassche T, Bosch J, Giruparajah M, Swaminathan $B$, Mattina KR, et al. Embolic strokes of undetermined source: prevalence and patient features in the ESUS Global Registry. Int J Stroke 2016;11:526-533.

23. Dewland TA, Olgin JE, Vittinghoff $E$, Marcus GM. Incident atrial fibrillation among Asians, Hispanics, blacks, and whites. Circulation 2013;128:2470-2477.

24. Zhou Z, Hu D. An epidemiological study on the prevalence of atrial fibrillation in the Chinese population of mainland China. J Epidemio/ 2008;18:209-216.

25. Kishore A, Vail A, Majid A, Dawson J, Lees KR, Tyrrell PJ, et al. Detection of atrial fibrillation after ischemic stroke or transient ischemic attack: a systematic review and meta-analysis. Stroke 2014;45:520-526.

26. Poulsen MB, Binici $Z$, Dominguez $H$, Soja AM, Kruuse $C$, Hornnes $A H$, et al. Performance of short ECG recordings twice daily to detect paroxysmal atrial fibrillation in stroke and transient ischemic attack patients. Int J Stroke 2017;12:192-196.
27. Zhang J, Yang XA, Zhang Y, Wei JY, Yang F, Gao H, et al. Oral anticoagulant use in atrial fibrillation-associated ischemic stroke: a retrospective, multicenter survey in Northwestern China. J Stroke Cerebrovasc Dis 2017;26:125-131.

28. Pandya EY, Anderson E, Chow C, Wang Y, Bajorek B. Contemporary utilization of antithrombotic therapy for stroke prevention in patients with atrial fibrillation: an audit in an Australian hospital setting. Ther Adv Drug Saf 2018;9:97-111.

29. Tanislav C, Allendörfer J, Pfeilschifter W, Fuest $S$, Stein $M$, Meyer $M$, et al. One decade of oral anticoagulation in stroke patients: results from a large country-wide hospital-based registry. Int J Stroke 2018;13:308-312.

30. Chang SS, Dong JZ, Ma CS, Du X, Wu JH, Tang RB, et al. Current status and time trends of oral anticoagulation use among Chinese patients with nonvalvular atrial fibrillation: the Chinese Atrial Fibrillation Registry Study. Stroke 2016;47:18031810.

31. Yang $X, L i Z$, Zhao $X$, Wang $C$, Liu L, Wang $C$, et al. Use of warfarin at discharge among acute ischemic stroke patients with nonvalvular atrial fibrillation in China. Stroke 2016;47:464470. 